Pak. j. sci. ind. res. Ser. B: biol. sci. 201457 (1) 25-31

\title{
Enrichment of Soymeal Medium to Increase the Rapamycin Production by Streptomyces hygroscopicus
}

\author{
Abdel-Hamid Ali Hamdy, Essam Mohamed Ahmed*, Lotfy Abd El-Raouf Sallam and \\ Mohamed Abdelaziz Mohamed \\ Natural and Microbial Products Department, National Research Center, Dokki, Cairo, Egypt
}

(received April 11, 2013; revised October 29, 2013; accepted October 31, 2013)

\begin{abstract}
Research was carried out to study the improved and increased production of rapamycin by Streptomyces hygroscopicus with soymeal enriched media. Media containing soymeal produced rapamycin upto $82.89 \mathrm{mg} / \mathrm{L}$. The medium was enriched with different additives that can interfere with biosynthesis process. L-tyrosine supplementations led to noticeable increase in the rapamycin production to $112 \mathrm{mg} / \mathrm{L}$. However, the progress was achieved upon addition of the shikimic acid (precursor rapamycin moiety), where, it reached $160 \mathrm{mg} / \mathrm{L}$. The greatest increase was recorded after addition of calcium superphosphate $(\mathrm{CaP})$ and the production achieved $176 \mathrm{mg} / \mathrm{L}$. Other substances like vitamins and trace elements had either no or negative effects on the biosynthesis of rapamycin. The study also showed the ability of low concentrations of calcium phosphate to replace the expensive large amount of shikimic acid.
\end{abstract}

Keywords: Streptomyces hygroscopicus, rapamycin, immunosuppressants, soymeal

\section{Introduction}

Rapamycin is a potent immunosuppressant, anticancer and antifungal medicine. Rapamycin was early discovered in 1975 as an antifungal agent produced by Streptomyces hygroscopicus and has a great potency against Candida albicans (Vezina et al., 1975). Up to date, rapamycin was approved twice from American FDA, the first one in 1999 for its ability to prevent host rejection in kidney transplanting and the second was in 2003 for its use in drug eluting stent to prevent restenosis of coronary arteries following angioplasty (Vezina et al., 1975).

In the recent years extensive research aiming at exploring more rapamycin activities ascertained that it represents an unreal tapped resource of clinical activities and can afford a lot of in drug realm as multifunction medication. It is anti-inflammatory and reduces the expression of several genes related to inflammation. In addition, it has antiangionenic, antiplorefiration and antifibrotic (Nuhrenberg et al., 2005; Morris, 1992). Rapamycin protects against hypoxic damage in primary heart culture via $\mathrm{Na} / \mathrm{Ca}$ exchange activation (El-Ani et al., 2011). Also rapamycin found to play role in regulation of gastric hormones (Xu et al., 2010). Furthermore, rapamycin does not affect post-absorptive protein metabolism in human skeletal muscle (Dickinson et al., 2013).

*Author for correspondence; E-mail: eahmed 98@hotmail.com
There are some trials to use rapamycin in treatment of acute myeloid leukemia, retinal and choriodal vascular diseases (Recher et al., 2005). With the tremendous nature of rapamycin activities the demands on this drug would be increased in future. Therefore, research work is focussed to improve its productivity and to reduce the production costs. The cuurent study aims increament of the rapamycin production by S. hygroscopicus through enrichment of natural media containing soybean meal. The media was selected after comparison with other synthetic and natural media. Additives that could interfere with the rapamycin biosynthesis supplied and tested for their effects.

This is the first time for such results to be recorded and present findings may be helpful for economic production of Rapamycin.

\section{Materials and Methods}

Microorganisms. The strain Streptomyces hygroscopicus ATCC 29253, was the experimental organism throughout the current study. Candida albicans ATCC 10231 was used in bioassay.

The Rap producer, S. hygroscopicus ATCC 29253, was grown on slants of oat meal medium (contained oat meal, $20 \mathrm{~g} / \mathrm{L}$; agar, $20 \mathrm{~g} / \mathrm{L}$ and $\mathrm{pH} 7$ ) for 10 days at $28^{\circ} \mathrm{C}$ after which spores were collected by addition of $4 \mathrm{~mL}$ of $10 \%(\mathrm{v} / \mathrm{v})$ glycerol to each slant. Spore 
suspensions were then pooled together to get a suspension of $25.8 \times 10^{6}$ c.f.u. $/ \mathrm{mL}$ that was then dispensed in cryopreservation vials, each contained $1 \mathrm{~mL}$, and stored at $-20{ }^{\circ} \mathrm{C}$.

Culture media. A medium composed of soybean meal, $30 \mathrm{~g}$; glucose, $20 \mathrm{~g}$; $\left(\mathrm{NH}_{4}\right)_{2} \mathrm{SO}_{4}, 5 \mathrm{~g}$; $\mathrm{KH}_{2} \mathrm{PO}_{4}$, $5 \mathrm{~g}$ (Sehgal et al., 1975), was selected after comparison with other seven synthetic and natural media of different compositions. The components dissolved in tap water of $\mathrm{pH} 6$ was used.

Fermentation. The culture was initiated by propagating $1 \mathrm{~mL}$ of thawed spore suspension containing $25.8 \times 10^{6}$ c.f.u. in $50 \mathrm{~mL}$ medium contained in $250 \mathrm{~mL}$ Erlenmeyer flask. Then each Erlenmeyer flasks $(250 \mathrm{~mL})$ containing $50 \mathrm{~mL}$ fermentation medium inoculated with $3 \mathrm{~mL}$ of inoculum. The inoculated flasks were incubated at $25^{\circ} \mathrm{C} \pm 2$ and $150 \mathrm{rpm}$ for 7 days. The fifth day was the optimal, where, after all fermentations were allowed to run for only 5 days.

Growth estimation. The dry cell weight was determined by placing a $10 \mathrm{~mL}$ sample from the whole fermentation medium into a pre-weighed $15 \mathrm{~mL}$ tube and centrifuged at $3500 \mathrm{rpm}$ for $5 \mathrm{~min}$. The microbial residue in the tube was dried at $80^{\circ} \mathrm{C}$ for 2 days and then weighed. The growth yield expressed as gram dry weight per liter fermentation medium. Packed cell volume percentage was determined by placing a $3 \mathrm{~mL}$ sample of whole fermentation medium into $10 \mathrm{~mL}$ tube and centrifuged at $3500 \mathrm{rpm}$ for $5 \mathrm{~min}$. The percentage of packed cell volume to the total volume of the whole fermentation medium sample was the desired estimate of growth.

Analysis. Aliquots of $3 \mathrm{~mL}$ were taken and the microbial growth was separated by centrifuging at $3500 \mathrm{rpm}$ for $5 \mathrm{~min}$ and extracted twice by shaking with $3 \mathrm{~mL}$ methanol for $30 \mathrm{~min}$. The two extracts were pooled to be assayed for Rap concentration. Bioassay determination of Rap was achieved by paper-disc agar diffusion method as described by Sallam et al. (2010) and Kojima and Demain (1998). A sample of $20 \mu \mathrm{L}$ was analysed with HPLC (SYKAM apparatus equipped with Injector, S5111; Pumps (two pumps), S1122; Column Thermo controller, S4011; Degaser, S7505; Column, Phenomenex Gemini C18 (250x4.6 mm, $5 \mu$ ); Detector, Jasco UV-2070Plus; Software, Autochro-3000). The sample injected and eluant of acetonitrile: water 9:1 was pumped at the rate of $1 \mathrm{~mL} / \mathrm{min}$ in column controlled at $55^{\circ} \mathrm{C}$. Rap was assessed by absorbance at $\lambda 277 \mathrm{~nm}$.

\section{Results and Discussion}

Amino acids addition. Different amino acids were added individually in the concentration of $5 \mathrm{~g} / \mathrm{L}$. The results presented in Fig. 1 show that, addition of L-tyrosine accelerated the production to be $48 \%$ greater than in control sample, whereas, L-lysine neither enhanced nor suppressed the production. All the other added amino acids suppressed Rap biosynthesis by different degrees and the complete cessation was in case of L-cysteine. Similar results were obtained by L-histidine, DL-aspartic acid, L-arginine, L-glutamic acid, glycin, L-asparagine and L-cystine, while, sharp deleterious effect was observed in case of L-tryptophan, L-alanine and DL-methionine. Also, with addition of L-valine and L-proline, Rap titre was dropped down to be around the third of that in control sample.

Growth measurement revealed some significant features. There was some degree of correlation between growth of the producing strain and Rap biosynthesis. In most cases, amino acids negatively affected the growth as well as Rap biosynthesis. In addition, L-tyrosine, that has potentiated Rap biosynthesis, has also favoured the growth. With addition of L-proline, fermentation process showed the same profile like in control sample, the same growth and final $\mathrm{pH}$ of fermentation medium, yet it had remarkable decrease in Rap titre.

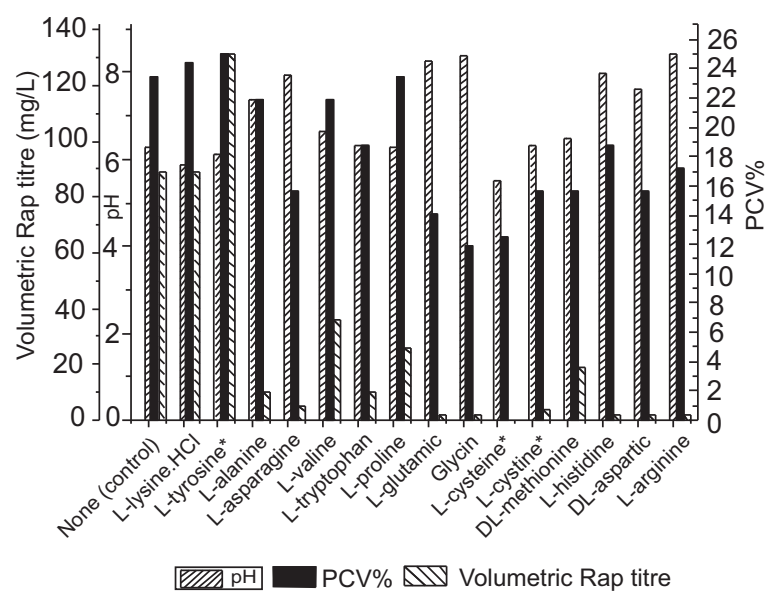

Fig. 1. Role of amino acids in biosynthesis of Rap by S. hygroscopicus ATCC 29253.

Biosynthesis of Rap in different augments of Ltyrosine. L-tyrosine was added to fermentation medium in different supplements namely; 1, 2, 5, 7.5, 10, 15, 20 and $30 \mathrm{~g} / \mathrm{L}$. The results that are represented in Fig. 2, show that decreasing L-tyrosine quantities to $2 \mathrm{~g} / \mathrm{L}$ can successfully afford the same promotion in 
Rap biosynthesis that was achieved at $5 \mathrm{~g} / \mathrm{L}$, and it yielded the best growth. More increase in L-tyrosine (more than $5 \mathrm{~g} / \mathrm{L}$ ) caused gradual depletion in Rap without effect on the microbial growth. The $\mathrm{pH}$ values remained constant at 6 .

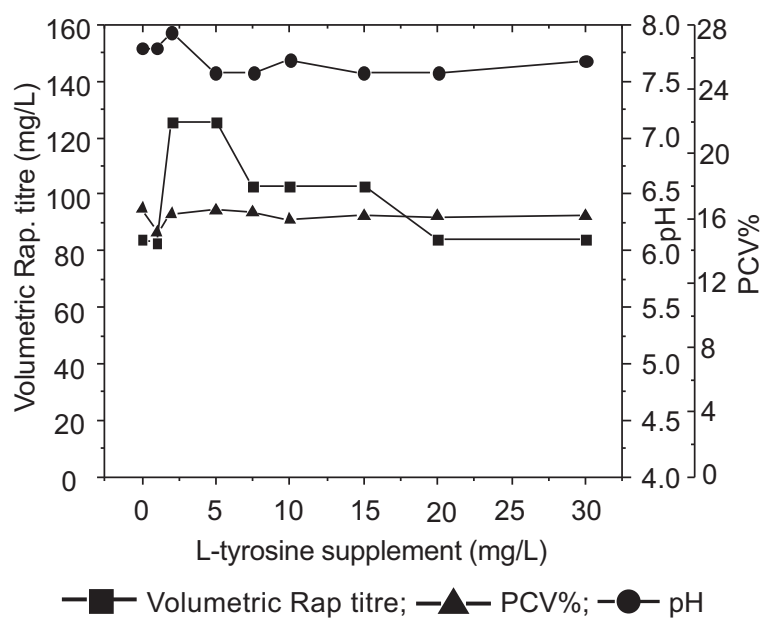

Fig. 2. Biosynthesis of Rap by S. hygroscopicus ATCC 29253 in different augments of L-tyrosine. PCV = packed cell volume.

Effect of time of L-tyrosine addition on Rap biosynthesis. According to the preceding investigations, L-tyrosine may play an obvious role in promoting Rap biosynthesis. In the current experiment, L-tyrosine $(2 \mathrm{~g} / \mathrm{L})$ was added at different times during fermentation. Time of L-tyrosine addition has a critical role in its promoting action (Table 1). The results revealed that L-tyrosine should be added at the start of fermentation and not after more than 1 day of fermentation. The effect of L-tyrosine could be inversed to remarkable suppression to the Rap biosynthesis as well as the growth, if it was added at the late stages of fermentation or at least after two days.

Effect of trace elements on Rap biosynthesis. With a production medium containing L-tyrosine $(2 \mathrm{~g} / \mathrm{L})$, trace elements were added, individually and in mixture, in concentrations like that were used by Lee et al. (1997). The data in Fig. 3 revealed that Rap biosynthesis was not favoured by addition of any of the studied trace elements. All of them have retarded Rap biosynthesis with only one exception in case of zinc salt, where, the results remained the same as that of control treatment. Copper ions (in very low concentration i.e., $1.3 \mathrm{mg} / \mathrm{L}$ ) exerted reduction in Rap to less than half of that in control sample. However, copper ion increased the
Table 1. Effect of time of L-tyrosine addition on Rap biosynthesis by Streptomyces hygroscopicus ATCC 29253

\begin{tabular}{llll}
\hline \hline $\begin{array}{l}\text { Time of L-tyrosine } \\
\text { addition (days) }\end{array}$ & $\begin{array}{l}\text { Final pH of } \\
\text { fermentation } \\
\text { medium }\end{array}$ & $\begin{array}{l}\text { Packed cell } \\
\text { volume } \\
(\mathrm{PCV} \%)\end{array}$ & $\begin{array}{l}\text { Volumetric } \\
\text { Rap titre } \\
(\mathrm{mg} / \mathrm{L})\end{array}$ \\
\hline Control* $_{0}$ & 6.30 & 25.94 & 82.87 \\
1 & 6.21 & 28.13 & 120.53 \\
2 & 6.23 & 28.13 & 120.53 \\
3 & 6.23 & 21.88 & 51.88 \\
4 & 6.23 & 22.18 & 37.97 \\
\hline \hline
\end{tabular}

* = no L-tyrosine addition.

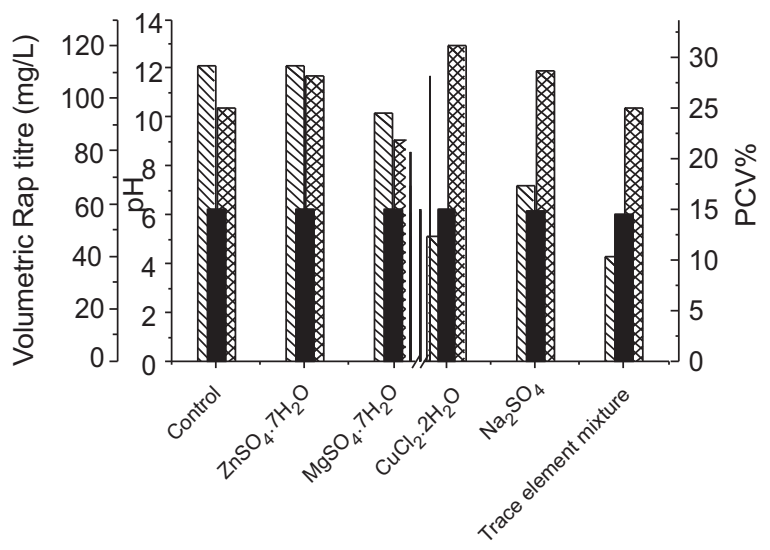

MIV Volumetric Rap titre;

$\mathrm{pH} \times$

Fig. 3. Effect of trace elements on Rap biosynthesis by S. hygroscopicus ATCC 29253. $\mathrm{PCV}=$ packed cell volume.

growth from 25 to $31 \% \mathrm{PCV}$. The same effect was also observed in case of boron, molybdenum and sodium formulated as $\mathrm{Na}_{2} \mathrm{~B}_{4} \mathrm{O}_{7},\left(\mathrm{NH}_{4}\right)_{6} \mathrm{MoO}_{24} \cdot 4 \mathrm{H}_{2} \mathrm{O}$ and anhydrous $\mathrm{Na}_{2} \mathrm{SO}_{4}$, respectively. Mixture of trace elements resulted in negative effect on Rap production higher than that with all individual trace elements. With all studied elements, limited variation in $\mathrm{pH}$ of fermentation medium was observed.

\section{Effect of addition of some vitamins on Rap bio-} synthesis. Different vitamins and vitamin precursors were added individually to the fermentation medium. Five milligrams of each vitamin were dissolved in $5 \mathrm{~mL}$ distilled water and the solution was then filtered and sterilised using syringe filter (of $0.45 \mu \mathrm{m}$ pore diameter) to be added under aseptic conditions to $250 \mathrm{~mL}$ Erlenmeyer flask containing $45 \mathrm{~mL}$ sterile medium. From the results in Fig. 4, it was obviously 
noted that Rap biosynthesis needs no medium enrichment with any of studied vitamins or vitamin precursors because none of them could increase Rap biosynthesis. On the contrary, only riboflavin had not hindered Rap biosynthesis, while, all the others exhibited inhibitory effect in different degrees. $p$-amino benzoic acid favoured the microbial growth. Remarkable suppression in Rap biosynthesis was observed with addition of biotin, pyridoxine and cyanocobalamine. The highest inhibition was in case of thiamine- $\mathrm{HCl}$, nicotinic acid and $\mathrm{L}(+)$ ascorbic acid.

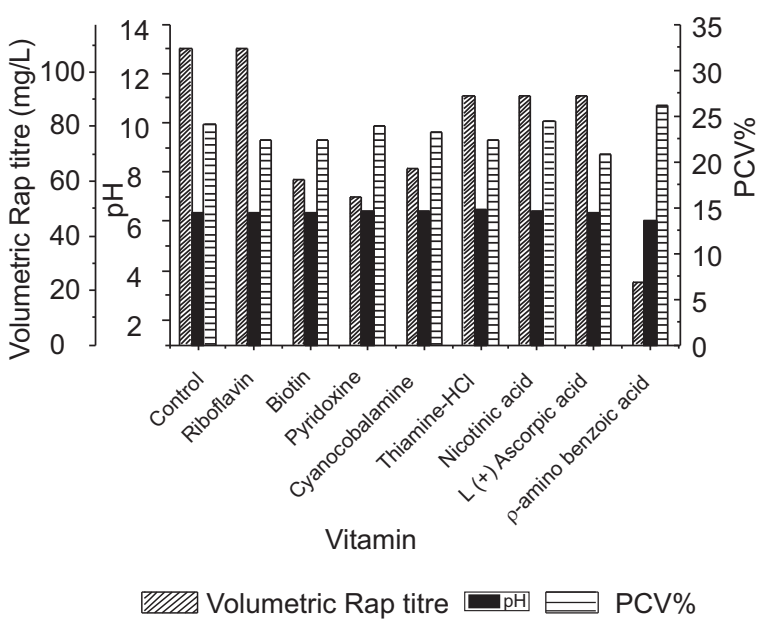

Fig. 4. Rap biosynthesis by S. hygroscopicus ATCC 29253 in media supplemented with different vitamins. $\mathrm{PCV}=$ packed cell volume.

\section{Rap biosynthesis in media enriched with precursors} and inducers. Knowing the building units and precursors involved in Rap biosynthesis justified the need to clarify the ability of these compounds to accelerate its production. The current experiment was developed to study the effect of adding acetate (building unit), shikimic acid (precursor of cyclohexane moiety in Rap) and calcium superphosphate $(\mathrm{CaP})$ on biosynthetic rate of Rap. Graphically reflected results demonstrated strong promotion in Rap production under the effect of shikimic acid and $\mathrm{CaP}$ addition (Fig. 5). More than 42\% achievement in Rap productivity was attained by addition of shikimic acid, which raised the titre from 112 to 160 $\mathrm{mg} / \mathrm{L}$. More close to that achievement was the increase from 112 to nearly $150 \mathrm{mg} / \mathrm{L}$ that had been attained with $\mathrm{CaP}$. Beside the strong promoting action of shikimic acid and $\mathrm{CaP}$ in Rap biosynthesis, they showed slight decrease in growth yield comparing with control sample.
On the other side, sodium acetate afforded complete suppression in Rap biosynthesis associated with very low PCV\%.

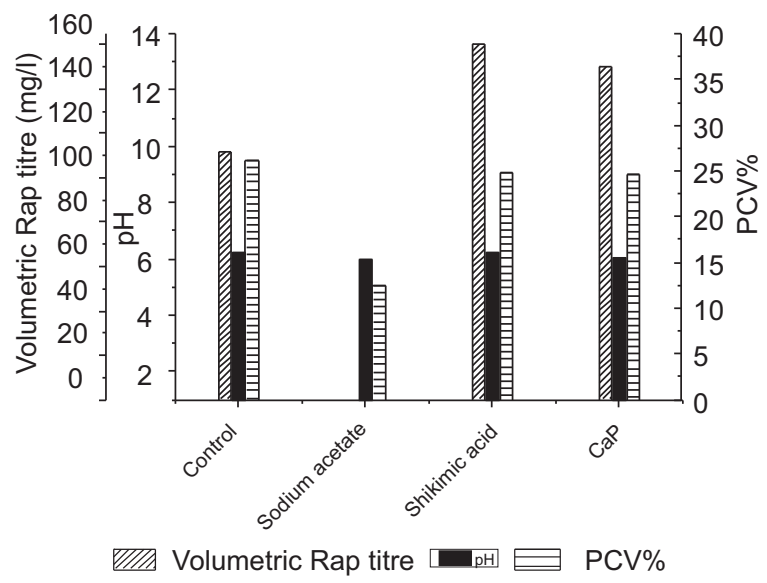

Fig. 5. Effect of medium enrichment with precursors and inducers on Rap biosynthesis by $S$. hygroscopicus ATCC 29253. CaP = calcium superphosphate; $\mathrm{PCV}=$ packed cell volume.

Rap biosynthesis in media enriched with different concentrations of shikimic acid and $\mathrm{CaP}$. This experiment was directed to follow up the Rap biosynthesis in presence of different concentrations of shikimic acid and $\mathrm{CaP}$ which applied in supplements ranging from 0.1 to $5 \mathrm{~g} / \mathrm{L}$. As indicated in Fig. 6, promotion in Rap biosynthesis attained by $5 \mathrm{~g} / \mathrm{L}$ shikimic acid could be achieved with lower concentration of $2 \mathrm{~g} / \mathrm{L}$. Shikimic acid was able to accelerate Rap production even at low concentration as $0.1 \mathrm{~g} / \mathrm{L}$. However, increasing its concentration to $0.7 \mathrm{~g} / \mathrm{L}$ was associated with non-significant increase in Rap titre. With little increase in its concentration from 0.7 to $1 \mathrm{~g} / \mathrm{L}$, shikimic acid exhibited considerable advance in Rap productivity. On the other side, the results in Fig. 7 revealed that $\mathrm{CaP}$ was also strong Rap stimulator when supplied in special amount. Interestingly, it was found that promoting action of $0.7 \mathrm{~g} / \mathrm{L} \mathrm{CaP}$ that was recorded in the former experiment could be intensified by lowering its concentration to $0.4 \mathrm{~g} / \mathrm{L}$. Comparing with control culture, Rap titre achieved more than $53 \%$ increase in cultures amended with $0.4 \mathrm{~g} / \mathrm{L} \mathrm{CaP}$. Concentration of $0.4 \mathrm{~g} / \mathrm{L}$ was the best for maximum acceleration in Rap biosynthesis and minor change upward or downward caused a drop in Rap production. When CaP concentration exceeded $1 \mathrm{~g} / \mathrm{L}$, final $\mathrm{pH}$ of fermentation began to move 


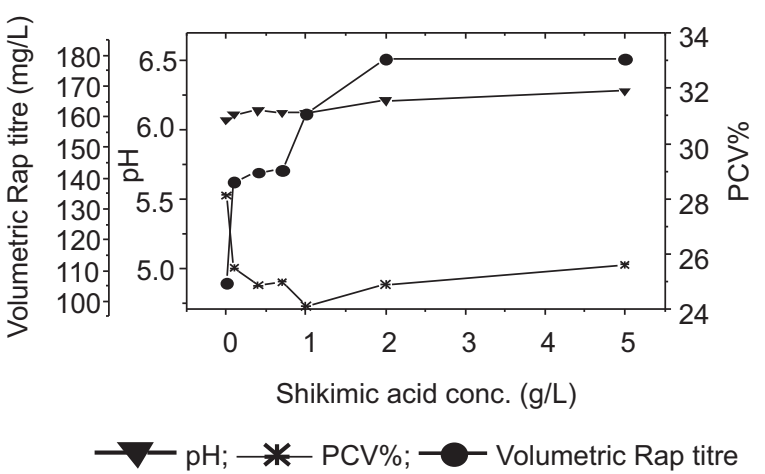

Fig. 6. Rap biosynthesis by S. hygroscopicus ATCC 29253 in media of different shikimic acid concentrations.

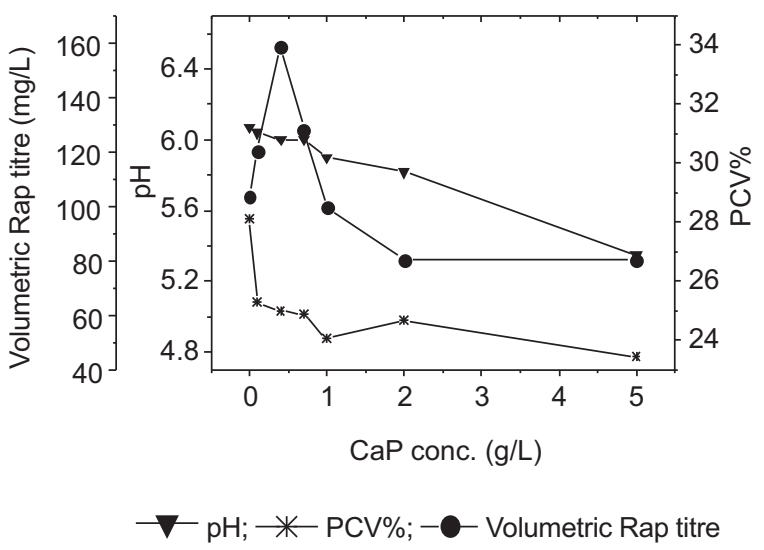

Fig. 7. Rap biosynthesis by S. hygroscopicus ATCC 29253 in media of different CaP concentrations.

belw 6 and Rap biosynthesis considerably decreased (Fig.7). The critical role of $\mathrm{CaP}$ in Rap biosynthesis, if well modulated, could be used to replace the promoting action mediated by high priced shikimic acid. In addition to its very low price comparing to shikimic acid, $\mathrm{CaP}$ works effectively in minor concentrations, which affords an extra advantage. The results indicated low growth yield in cultures amended with shikimic acid or $\mathrm{CaP}$ and this provides an industrial economic input.

Applying L-tyrosine at different amounts showed that each of 2 or $5 \mathrm{~g} / \mathrm{L}$ supplements could afford the same promoting action in Rap biosynthesis though $2 \mathrm{~g} / \mathrm{L}$ was the optimal for growth. Promoting action of L-tyrosine was attained only, when it was added at start of fermentation or at most after one day lapse. At the second day of fermentation, addition of
L-tyrosine gave rise to severe drop in Rap titre (51.88 $\mathrm{mg} / \mathrm{L}$ ), which was lower than that in tyrosine free culture $(82.87 \mathrm{mg} / \mathrm{L})$. It was proposed that incorporating tyrosine after time lapse of fermentation stimulates the metabolism to direct towards L-tyrosine, which may interrupt already working metabolic activities, and cells would spend some lagging period before being adapted to metabolise tyrosine. The lost time is reflected as a reduction in growth and Rap titer as it has shown in the current results.

Various metals affect the production of antibiotics (Iwai and Omura, 1982). The results showed that Rap biosynthesis was not favoured by any of studied trace elements. Copper ions (in very low concentration of $1.3 \mathrm{mg} / \mathrm{L}$ ) exerted reduction in Rap titre to less than half of that in control sample together with increasing growth from 25 to $31 \%$ PCV. With all studied trace elements, limited variation in $\mathrm{pH}$ of fermentation medium was shown indicating that variation in outputs of Rap and growth was not attributed to shift in medium $\mathrm{pH}$.

Enriching fermentation medium with different vitamins and vitamin precursors elucidated the retarding effect. $\rho$-amino benzoic acid caused the greatest drop in Rap titre and in the same time it had favoured the microbial growth which reflected the difference between requirements needed for growth and Rap biosynthesis. The role of vitamins in Rap biosynthesis may better be investigated in chemically defined medium. It is important to note that soy meal, a medium component, contains self constituents of vitamins (USDA National Nutrient database). The negative action in the obtained results may be attributed to rise in vitamin concentration after exogenous vitamin supplementations.

Shikimic acid and acetate are known precursors in Rap biosynthesis (Reynolds and Demain, 1997). The results of the current work showed strong promotion in Rap biosynthesis under the effect of shikimic acid addition. Also, calcium superphosphate $(\mathrm{CaP})$ has potentiated comparable promotion. Beside the strong promoting action of shikimic acid and $\mathrm{CaP}$ in Rap biosynthesis, they showed slight decrease in growth yield comparing with control sample. These findings may point to a specific positive interaction of shikimic acid and $\mathrm{CaP}$ with metabolic activities incorporated in Rap biosynthesis. Decrease in growth yield may be result of 
stimulatory effect of these precursors that initiate Rap biosynthesis earlier after short time of growth proliferation. Low growth yield may provide an advantage due to conserved downstream processing and extracting Rap from less biomass yield. Although Fang and Demain (1995) have elucidated the stimulatory action of shikimic acid on Rap production in chemically defined medium, they failed to record its promotion in complex medium. As such, stimulatory action of shikimic acid in a complex medium was reported here for the first time. Relatively low concentration $(0.7 \mathrm{~g} / \mathrm{L})$ could markedly induce Rap biosynthesis greater than $5 \mathrm{~g} / \mathrm{L}$ shikimic acid. Calcium superphosphate may need extensive future studies to resolve such role. Omura et al. (1980) reported the tremendous promotion, 2-15 times, in production of spiramycin, a macrolide antibiotic, by Streptomyces ainbofaciens ATCC 23877 after $\mathrm{CaP}$ addition. Thus, the inductive action of $\mathrm{CaP}$ in biosynthesis of Rap, as one of the most valuable recent drugs, was recorded here for the first time. Complete suppression of Rap biosynthesis, very low $\mathrm{PCV} \%$ value and non changed medium $\mathrm{pH}$, in case of fermentation amended with sodium acetate makes the suggestion that sodium acetate has strong toxic effect on the experimental organism. It was found that shikimic acid concentration of $2 \mathrm{~g} / \mathrm{L}$ has the same effect of 5 $\mathrm{g} / \mathrm{L}$, where about $176 \mathrm{mg} / \mathrm{L}$ of Rap was obtained and the effect of $\mathrm{CaP}$ was found to be intensified by $53 \%$ by lowering its concentration from 0.7 to $0.4 \mathrm{~g} / \mathrm{L}$. Another feature in $\mathrm{CaP}$ stimulatory function; when $\mathrm{CaP}$ concentration exceeded $1 \mathrm{~g} / \mathrm{L}$, the final $\mathrm{pH}$ was decreased and Rap biosynthesis was delayed. It may be assumed that relatively higher $\mathrm{CaP}$ concentrations (nearly more than $1 \mathrm{~g} / \mathrm{L}$ ) alter the fermentation medium $\mathrm{pH}$, which decreases the Rap production. Care should be taken to select the suitable buffer of non-interference with metabolic activities of the experimental organism. Thus, replacement of $2 \mathrm{~g} / \mathrm{L}$ shikimic acids which is expensive substance, with $0.4 \mathrm{~g} / \mathrm{L}$ of cheap calcium superphosphate will be of economic value.

\section{References}

Dickinson, M.J., Drummound, J.M., Fry, S.C., Gundermann, M.D., Walker, K.D., Timmermann, L.K., Volpi, E., Rosmussen, B.B. 2013. Rapamycin does not affect post-absorptive protein metabolism in human skeletal muscle. Metabolism: Clinical and Experimental, 62: 144-151.

El-Ani, D., Stave, H., Guetta, V., Arad, M., Shaiberg, A. 2011. Rapamycin (sirolimus) protects against hypoxic damage in primary heart culture $v i a \mathrm{Na} / \mathrm{Ca}$ exchange activation. Life Science, 89: 7-14.

Fang, A., Demain, A.L. 1995. Exogenous shikimic acid stimulates rapamycin biosynthesis in Streptomyces hygroscopicus. Folia Microbiology, 40: 607-610.

Iwai, Y., Omura, S. 1982. Culture conditions for screening of new antibiotics. Journal of Antibiotics, 35: 123-141.

Kojima, I., Demain, A.L. 1998. Preferential production of Rapamycin vs prolylrapamycin by Streptomyces hygroscopicus. Journal of Industrial Microbiology and Biotechnology, 20: 309-316.

Lee, M.S., Kojima, I., Demain, A.L. 1997. Effect of nitrogen source on biosynthesis of rapamycin by Streptomyces hygroscopicus. Journal of Industrial Microbiology and Biotechnology, 19: 83-86

Morris, R.E. 1992. Rapamycins: antifungal, antitumor, antiproliferative and immunosuppressive macrolides. Transplantation Reviews, 16: 39-87.

Nuhrenberg, T.G., Voisard, R., Fahlisch, F., Rudelius, M., Braun, J., Gschwend, J., Kountides, M., Herter, T., Baur, R., Hombach, V., Baeuerle, P.A., Zohlnhofer, D. 2005. Rapamycin attenuates vascular wall inflammation and progenitor cell promoters after angioplasty. FASEB Journal, 19: 246-248.

Omura, S., Tanaka, Y., Tanaka, H., Takahashi, Y., Iwai, Y. 1980. Stimulation of the production of macrolide antibiotics by magnesium phosphate and related insoluble materials. Journal of Antibiotic, 33: 16581659.

Recher, C., Beyne-Rauzy, O., Demur, C., Chicanne, G., Santos, C.D., Mas, V.M., Benzaquen, D., Laurent, G., Huguet, F., Payrastre, B. 2005. Antileukemic activity of rapamycin in acute myeloid leukemia. Blood, 105: 2527-2534.

Reynolds, K.A., Demain, A.L. 1997. Rapamycin, FK506 and ascomycin-related compounds. In: Biotechnology of Antibiotics, W. R. Strohled (ed.), pp. 497-520, $2^{\text {nd }}$ edition, Marcel Dekker, New York, 
USA.

Sallam, L.A.R., El-Refai, A.F., Osman, M.E., Hamdy, A.A., Ahmed, E.M., Mohamed, M.A. 2010. Some physiological factors affecting rapamycin production by Streptomyces hygroscopicus ATCC 29253. Journal of American Science, 6: 188-194.

Sehgal, S., Baker, H., Vezina, C. 1975. Rapamycin (AY22,989), a new antifungal antibiotic. II. Fermentation, isolation and characterization. Journal of Antibiotics, 28: 727-732.
Vezina, C., Kudelski, A., Sehgal, S.N. 1975. Rapamycin (AY-22,989), a new antifungal antibiotic. I. Taxonomy of the producing streptomycete and isolation of the active principle. Journal of Antibiotics, 28: 721-726.

Xu, G., Li, Y., An, W., Zhao, J., Xiang, X., Ding, L., Guan, Y., Wang, X., Tang, C., Zhu, Y., Wang, N., Xiaoying, L., Mulholland, N., Zhang, W. 2010. Regulation of gastric hormones by systemic rapamycin. Peptide, 3: 2185-2192. 